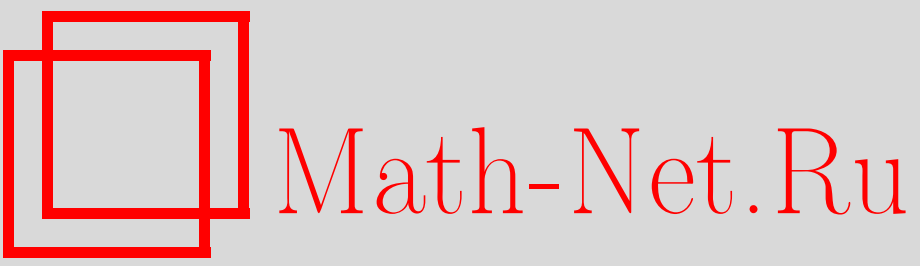

Т. А. Аракелян, Когомологическая структура конформной аномалии, ТМФ, 1998, том 117, номер 3, 351-363

DOI: https://doi.org/10.4213/tmf937

Использование Общероссийского математического портала Math-Net.Ru подразумевает, что вы прочитали и согласны с пользовательским соглашением

http://www.mathnet.ru/rus/agreement

Параметры загрузки:

IP: 54.166 .219 .16

26 апреля 2023 г., 13:39:57 
ТЕОРЕТИЧЕСКАЯ

И МАТЕМАТИЧЕСКАЯ

ФИЗИКА

Том 117, № 3

декабрь, 1998

(C) 1998 г.

Т. А. Аракелян*

\title{
КОГОМОЛОГИЧЕСКАЯ СТРУКТУРА КОНФОРМНОЙ АНОМАЛИИ
}

\begin{abstract}
Рассматривается когомологическая классификация разных видов конформных аномалий в размерности $D=6$. Показано, что с точки зрения когомологической интерпретации разные классы этих аномалий имеют общее происхождение. Именно все они эквивалентны плотности Эйлера $E_{6}$. По аналогии с калибровочными теориями развивается техника уравнения спуска для конформных аномалий. Исследуются все высшие коциклы группы Вейля. Описан обший метод построения всех конформных аномалий из плотности Эйлера $E_{2 n}$ в произвольных размерностях пространства-времени. Показано принципиальное различие структур этих аномалий в размерностях $D=4$ и $D=6$. Доказано отсутствие при размерности $D=6$ конформно-инвариантного оператора (построенного из тензоров Римана, Риччи, скалярной кривизны и ковариантных производных), действующего на скаляр с нулевым конформным весом.
\end{abstract}

\section{1. ВВЕДЕНИЕ}

Исторически конформная аномалия описана более тридцати лет назад Даффом в его фундаментальных работах $[1,2]$. Вопрос о конформной аномалии имеет длинную историю и многочисленные приложения в разных областях теоретической физики. Конформная симметрия играет первостепенную роль в теории струны и конформной теории поля. Эта симметрия была исследована с разных точек зрения как на классическом, так и на квантовом уровнях. Тем не менее полное понимание принципа нарушения конформной симметрии на квантовом уровне в высших размерностях (обязательно четномерных) отсутствует.

Последнее время, в основном благодаря работе Дезера и Швиммера [2], возобновляется интерес к проблеме классификаций конформных аномалий в произвольных размерностях. Геометрическая классификация конформной аномалии, предложенная этими авторами, основана на масштабных свойствах эффективного гравитационного действия, которое индуцируется конформно-инвариантными полями материи (спинорными, скалярными, векторными и т.д.). Вместе с тем Бонора и др. в серии работ $[3,4]$ по аналогии с калибровочными и гравитационными аномалиями исследовали когомологическую структуру конформной аномалии. Когомологический подход оказывается более успешным, поскольку дает возможность получить для аномалий самосогласованные

\footnotetext{
*Ереванский физический институт, Ереван, Армения
} 
выражения (удовлетворяющие условию Весса-Зумино) в высших размерностях пространства-времени без использования эффективного гравитационного действия. Хорошо известно, что вычисления, используюшие эффективное гравитационное действие, основаны на теории возмущения и конкретных схемах регуляризации для устранения расходимостей [1], в то время как подход, основанный на исследовании когомологических классов группы Вейля, более универсален и не зависит от теории возмушения и схем регуляризаций [4-7].

В этой работе мы продолжаем когомологический анализ конформных аномалий в высших размерностях, используя технику уравнений спуска. Мы строим также высшие коциклы для разных типов аномалий и классифицируем их с точки зрения когомологического подхода. Кроме того, строится новый тип скаляра при размерности $D=6$, инвариантного относительно преобразований Вейля. Этот скаляр состоит из двух тензоров Вейля, двух ковариантных производных и отличается от известных выражений для двух скаляров, построенных из трех тензоров Вейля. Мы предполагаем, что такие инварианты существуют также и для высших размерностей. Общее доказательство когомологической эквивалентности аномалий типов (А) и (В) [2] основано на сушествовании таких инвариантов.

Работа организована следующим образом. Во втором разделе описаны возникновение конформной аномалии и условие самосогласованности, которому она удовлетворяет. Строится нильпотентный оператор $\delta$ на калибровочной группе (Вейля), определяются коцепи на группе и когомологические классы когранищ [6]. Вводится другой нильпотентный оператор $\widetilde{\nabla}_{\mu}$, действуюший на полностью антисимметричных плотностях (аналог внешнего дифференцирования для форм). Вводится концепция уравнений спуска для классификации аномалий и инвариантных лагранжианов. В разделе 3 на этой основе классифицируются конформные аномалии в размерности $D=6$, выводится новый тип аномалий. Из когомологической эквивалентности аномалий типов $(\mathrm{A})$ и $(\mathrm{B})$ найдено выражение, инвариантное относительно групшы Вейля. Дается явное выражение для высших коциклов группы Вейля. В разделе 4 исследуется возможность построения конформно-инвариантного оператора 6-го порядка (включаюшего оператор $\Delta^{3}$ как слагаемое), доказана теорема об отсутствии таких операторов в размерности $D=6$. Заметим, что для размерности $D=4$ сушествование таких операторов хорошо известно [8]. В разделе 5 обсуждается различие структур конформных аномалий в размерностях $D=4$ и $D=6$, предлагаются различные способы преодоления трудностей, возникаюших при построении эффективного гравитационного действия в размерности $D=6$.

\section{2. УРАВНЕНИЯ СПУСКА ДЛЯ КОНФОРМНЫХ АНОМАЛИЙ}

Рассмотрим поля материи, взаимодействуюшие с внешним гравитационным полем. Действие $S_{\mathrm{W}}(\varphi, g)$, описываюшее эту систему, инвариантно относительно группы Вейля и диффеоморфизмов 6-мерного пространства-времени. Эффективное гравитационное действие получается при интегрировании по полям материи и имеет вид

$$
W[g]=\ln \int \mathcal{D}_{g} \varphi \exp \left\{-S_{\mathrm{W}}(\varphi, g)\right\}
$$


Утверждение о существовании конформной аномалии заключается в нарушении конформной инвариантности $W[g]$ относительно преобразования Вейля метрики

$$
g_{\alpha \beta} \rightarrow \hat{g}_{\alpha \beta}=\exp \{\Omega(x)\} g_{\alpha \beta}(x) .
$$

Тогда аномальное тождество Уорда принимает вид

$$
\delta W[g]=\int d^{6} x \sqrt{g(x)} T_{\mu}^{\mu}(x) \delta \Omega(x) .
$$

Хорошо известно, что функциональная зависимость аномалий от метрики не может быть произвольной, а должна удовлетворять условию самосогласованности Весса-Зумино [5-7]. Последнее условие эквивалентно симметричности второй вариации $W[g]$ :

$$
\frac{\delta^{2} W[g]}{\delta \Omega(x) \delta \Omega(y)}=\frac{\delta^{2} W[g]}{\delta \Omega(y) \delta \Omega(x)}
$$

Здесь необходимо сделать несколько замечаний относительно конформной аномалии. Из теории возмушений для аномалии в следе вытекает нелокальный характер эффективного действия, а из этого факта - необходимость изменения обшепринятого определения эквивалентности для двух типов аномалий. Условию самосогласованности Весса-Зумино можно придать вид

$$
\frac{\delta\left[\sqrt{g(x)} T_{\mu}^{\mu}(x)\right]}{\delta \Omega(y)}=\frac{\delta\left[\sqrt{g(y)} T_{\mu}^{\mu}(y)\right]}{\delta \Omega(x)} .
$$

Интегрируя по переменным $x$ и $y$, имеем

$$
\int d^{6} y \frac{\delta L[g]}{\delta \Omega(y)}=\int d^{6} x \frac{\delta L[g]}{\delta \Omega(x)}
$$

где

$$
L[g]=\int d^{6} x \sqrt{g(x)} T_{\mu}^{\mu}(x) .
$$

В такой форме для $L[g]$ условие Весса-Зумино всегда выполняется, поскольку в результате интегрирования в $(7)$ вариация $\delta\left(\sqrt{g} T_{\mu}^{\mu}\right)$ определена с точностью до полной производной. Следовательно, две аномалии мы понимаем как эквивалентные, если конформные вариации отличаются на полную производную. Вспомним, что в калибровочных и гравитационных теориях две аномалии эквивалентны, если

$$
\widetilde{\omega}_{n}^{1}-\omega_{n}^{1}=\delta \Omega_{n}+d \sum_{n-1}^{1} .
$$

Таким образом, наше определение эквивалентности - более слабое требование, чем это условие. Оказывается, существует удобньй и красивый формализм, позволяющий чисто алгебраическим путем решить проблему классификаций инвариантных лагранжианов и аномалий. Для демонстрации этого формализма введем следуюший оператор 
конформной вариации, действующий на пространстве полиномов и построенный из тензоров Римана и ковариантных производных

$$
\begin{aligned}
\delta \hat{g}_{\alpha \beta} & =\delta \Omega \cdot \hat{g}_{\alpha \beta}, \quad \delta(\delta \Omega)=0, \\
\delta^{2} \hat{g}_{\alpha \beta} & =\delta(\delta \Omega) \hat{g}_{\alpha \beta}+(\delta \Omega)^{2} \hat{g}_{\alpha \beta}=0
\end{aligned}
$$

Оператор $\delta$, действуя на 0-коцепях, отображает их на 1-коцепи группы Вейля $(D=6)$ :

$$
\begin{aligned}
\delta \Gamma_{\rho \nu}^{\lambda} & =\frac{1}{2}\left(\delta_{\rho}^{\lambda} \nabla_{\nu} \delta \Omega+\delta_{\nu}^{\lambda} \nabla_{\rho} \delta \Omega-g_{\nu \rho} \nabla^{\lambda} \delta \Omega\right) \\
\delta R_{\rho \sigma \nu \mu} & =\delta \Omega R_{\rho \sigma \nu \mu}+\frac{1}{2}\left(g_{\nu \rho} \nabla_{\sigma} \nabla_{\mu} \delta \Omega+g_{\sigma \mu} \nabla_{\nu} \nabla_{\rho} \delta \Omega-(\nu \rightleftarrows \mu)\right) .
\end{aligned}
$$

Из этих формул следует, что

$$
\begin{aligned}
\delta R_{\sigma \mu} & =\frac{1}{2}\left(4 \nabla_{\sigma} \nabla_{\mu} \delta \Omega+g_{\sigma \mu} \Delta \delta \Omega\right) \\
\delta R & =-\delta \Omega \cdot R+5 \Delta \delta \Omega
\end{aligned}
$$

Правила коммутации $\delta$ и $\nabla_{\lambda}$ даются формулой

$$
\delta \nabla_{\alpha} A_{\mu_{1} \ldots \mu_{n}}=\nabla_{\alpha} \delta A_{\mu_{1} \ldots \mu_{n}}-\delta \Gamma_{\alpha \mu_{1}}^{\sigma} A_{\sigma \ldots \mu_{n}}-\cdots-\delta \Gamma_{\alpha \mu_{n}}^{\sigma} A_{\mu_{1} \ldots \sigma}
$$

Для дальнейших вычислений будет полезно ввести следуюшие определения для полиномов, построенных из тензоров Римана. Обозначим через $R_{\left\{\lambda_{1}, \ldots, \lambda_{q}\right\}}^{r}$ множество всех полиномов от тензоров Римана ранга $r$ (количество свободных индексов), степени $q$ (число множителей $\nabla^{k} R$ ) и порядка $S$ (число дифференцирования метрики), которые имеют следуюший вид:

$$
\nabla \cdot \ldots \nabla \cdot R_{. .}^{\cdot} \nabla^{\cdot} \ldots \nabla^{\cdot} R_{. .}^{.} \ldots \nabla^{\cdot} \ldots \nabla^{\cdot} R_{. .}^{.}
$$

где рассматриваются всевозможные свертки индексов, кроме $r$ штук, которые остаются свободными, и

$$
\sum_{i=1}^{q} \lambda_{i}+2 q=S
$$

Обозначим через $\omega_{\left\{k_{1}, \ldots, k_{p}\right\}}^{l}$ пространство всех элементов вида

$$
\nabla \cdot \ldots \nabla \cdot \delta \Omega \ldots \nabla \cdot \ldots \nabla \cdot \delta \Omega
$$

c $l$ свободными индексами. Коцепь порядка $p$ состоит из суммы произведений элементов из $\omega_{\left\{k_{1}, \ldots, k_{p}\right\}}^{l}$ и $R_{\left\{\lambda_{1}, \ldots, \lambda_{q}\right\}}^{r}$ со всевозможными свертками свободных индексов. Таким образом, символически можно написать

$$
\begin{aligned}
C_{\left\{k_{1}, \ldots, k_{p}\right\}} & =\bigoplus_{m=0}^{r+l} C_{\left\{k_{1}, \ldots, k_{p}\right\}}^{m}, \\
C_{\left\{k_{1}, \ldots, k_{p}\right\}}^{m} & =\bigoplus_{\lambda_{i} \geqslant 0} C\left(R_{\left\{\lambda_{1}, \ldots, \lambda_{q}\right\}}^{r} \omega_{\left\{k_{1}, \ldots, k_{p}\right\}}^{l}\right),
\end{aligned}
$$


где $C$ перед скобкой означает свертку $r+l$ свободных индексов до $m$-го. Такое определение понадобится для рассмотрения различных коцепей, возникаюших в уравнении спуска. Заметим, что два элемента, отличающихся расположением ковариантных производных в мономах вида $\nabla \cdot \ldots \nabla \cdot R .$. , предполагаются эквивалентными. Это следует из тождества

$$
\left[\nabla_{\mu}, \nabla_{\nu}\right] A_{\lambda}=-R_{\mu \nu}^{\rho}{ }_{\lambda} A_{\rho}
$$

и того факта, что разность этих элементов принадлежит пространству $R_{\left\{\lambda_{1}, \ldots, \lambda_{q+1}\right\}}^{\mu}$. То же самое относится и к элементам из пространства $\omega_{\left\{k_{1}, \ldots, k_{p}\right\}}^{l}$. Разность таких элементов принадлежит пространству $C_{\left\{k_{1}, \ldots, k_{p}\right\}}^{m}$. Теперь мы можем определить правила коммутации для коцепей и операторов $\delta$. Как мономы $\nabla^{k_{1}} \delta \Omega$ и $\nabla^{k_{2}} \delta \Omega$, так и операторы $\delta$ и $\nabla^{k} \delta \Omega$ антикоммутативны. Из этих определений немедленно получаем, что

$$
\begin{aligned}
C_{p} C_{q} & =(-1)^{p q} C_{q} C_{p}, \\
\delta\left(C_{p} C_{q}\right) & =\delta C_{p} \cdot C_{q}+(-1)^{p} C_{p} \cdot \delta C_{q} .
\end{aligned}
$$

Из соотношения (10) следует, что на 0-коцепях $\delta^{2}=0$. Из (17) имеем нильпотентность оператора $\delta$ на произвольных коцепях. Это свойство дает возможность определить когомологические классы оператора $\delta$, т.е.

$$
H_{p}=\frac{\left(\operatorname{Ker} \delta_{p}\right)}{\operatorname{Im} \delta_{p-1}}
$$

где нижний индекс $p$ у оператора показьвает, что последний действует на коцепях порядка $p$ и тем самым определяет $p$-ю группу когомологий. Мы также введем оператор внешнего дифференцирования, действуюший на полностью антисимметричные плотности, которые одновременно являются коцепями порядка $P$ :

$$
\begin{aligned}
A_{p}^{\mu_{1} \ldots \mu_{n}} & =\sqrt{g} C_{p}^{\mu_{1} \cdots \mu_{n}}, \\
C_{p}^{\mu_{\pi(1)} \ldots \mu_{\pi(n)}} & =(-1)^{N_{\pi}} C_{p}^{\mu_{1} \ldots \mu_{n}}, \\
\widetilde{\nabla}_{\mu_{1}}: A_{p}^{\mu_{1} \ldots \mu_{n}} & \rightarrow A_{p}^{\mu_{2} \ldots \mu_{n}}, \\
\widetilde{\nabla}_{\mu_{1}} A_{p}^{\mu_{1} \ldots \mu_{n}} & =\sqrt{g} \nabla_{\mu_{1}} \frac{1}{\sqrt{g}} A_{p}^{\mu_{1} \mu_{2} \ldots \mu_{n}} .
\end{aligned}
$$

Для полностью антисимметричных тензоров известна следующая формула:

$$
\nabla_{\mu_{1}} C_{p}^{\mu_{1} \mu_{2} \ldots \mu_{n}}=\frac{1}{\sqrt{g}} \partial_{\mu_{1}}\left(\sqrt{g} C_{p}^{\mu_{1} \mu_{2} \ldots \mu_{n}}\right),
$$

которая показывает, что оператор $\widetilde{\nabla}_{\mu_{1}}$ действует как обычная производная

$$
\widetilde{\nabla}_{\mu_{1}} A_{p}^{\mu_{1} \mu_{2} \ldots \mu_{n}}=\partial_{\mu_{1}} A_{p}^{\mu_{1} \mu_{2} \ldots \mu_{n}}=\sqrt{g} \nabla_{\mu_{1}} C_{p}^{\mu_{1} \mu_{2} \ldots \mu_{n}} .
$$

Такое определение удобно для сохранения общекоординатной инвариантности плотностей. Вследствие этого постулируем коммутативность $\delta$ и $\widetilde{\nabla}_{\mu}$ :

$$
\left[\delta, \widetilde{\nabla}_{\mu}\right]=0 .
$$


Из этих свойств оператора $\widetilde{\nabla}_{\mu}$ следует его нильпотентность $\left(\widetilde{\nabla}_{\mu}\right)^{2}=0$ :

$$
\widetilde{\nabla}_{\mu_{2}} \nabla_{\mu_{1}} A_{p}^{\mu_{1} \mu_{2} \ldots \mu_{n}}=\sqrt{g} \nabla_{\mu_{2}} \nabla_{\mu_{1}} C_{p}^{\mu_{1} \mu_{2} \ldots \mu_{n}}=\partial_{\mu_{2}} \partial_{\mu_{1}} A_{p}^{\mu_{1} \mu_{2} \ldots \mu_{n}}=0 .
$$

Теперь можно переформулировать условие самосогласованности Весса-Зумино для эффективного действия на когомологическом языке:

$$
\begin{aligned}
\delta^{2} W[g] & =0, \\
\delta\left(\sqrt{g} T_{\mu}^{\mu}(x)\right) & =\sqrt{g} \nabla_{\lambda}\left(A^{\lambda \rho} \nabla_{\rho} \delta \Omega\right)=\widetilde{\nabla}_{\lambda} A_{1}^{\lambda},
\end{aligned}
$$

где $A^{\lambda \rho}=A^{\rho \lambda}-$ симметричный по индексам полином от тензоров Римана. Соотношение (23) является следствием симметричности второй вариации $W[g]$ и антикоммутативности $\delta \Omega(x)$ и $\delta \Omega(y)$. В когомологическом подходе первое соотношение в $(23)$ - это просто следствие нильпотентности оператора $\delta$, в то время как второе - следствие условия, являюшегося первым соотношением уравнения спуска. Приведенные ниже соотношения следуют из (23), нильпотентности операторов $\delta$ и $\widetilde{\nabla}_{\mu}$ и алгебраического варианта леммы Пуанкаре:

$$
\begin{aligned}
\delta A_{1}^{\lambda} & =\widetilde{\nabla}_{\mu} A_{2}^{\lambda \mu}, \\
& \vdots \\
\delta A_{k-1}^{\mu_{1} \ldots \mu_{k-1}} & =\widetilde{\nabla}_{\mu_{k}} A_{k}^{\mu_{1} \ldots \mu_{k-1} \mu_{k}}, \\
\delta A_{k}^{\mu_{1} \ldots \mu_{k-1} \mu_{k}} & =0 .
\end{aligned}
$$

\section{3. ЭКВИВАЛЕНТНОСТЬ АНОМАЛИЙ В РАЗМЕРНОСТИ $D=6$}

Рассмотрим разные формы аномалий в размерности $D=6$, удовлетворяюшие условию самосогласованности Весса-Зумино. Как известно, существует аномалия типа (A) [2], пропорциональная плотности Эйлера $E_{6}$. Наша цель заключается в получении уравнения спуска для аномалий такого типа:

$$
\begin{aligned}
\delta E_{6} & =\widetilde{\nabla}_{\mu} B_{1}^{\mu}, \\
\delta B_{1}^{\mu} & =\widetilde{\nabla}_{\nu} B_{2}^{\mu \nu}, \\
\delta B_{2}^{\mu \nu} & =\widetilde{\nabla}_{\rho} B_{3}^{\mu \nu \rho}, \\
\delta B_{3}^{\mu \nu \rho} & =0
\end{aligned}
$$

где

$$
\begin{aligned}
E_{6} & =\sqrt{g} \varepsilon^{\alpha_{1} \ldots \alpha_{6}} \varepsilon_{\beta_{1} \ldots \beta_{6}} R_{\alpha_{1} \alpha_{2}}^{\beta_{1} \beta_{2}} R_{\alpha_{3} \alpha_{4}}^{\beta_{3} \beta_{4}} R_{\alpha_{5} \alpha_{6}}^{\beta_{5} \beta_{6}}, \\
B_{1}^{\mu} & =-6 \sqrt{g} \varepsilon^{\mu \alpha_{2} \ldots \alpha_{6}} \varepsilon_{\beta_{1} \ldots \beta_{6}} \delta \Gamma_{\gamma \alpha_{2}}^{\beta_{1}} \cdot g^{\gamma \beta_{2}} R_{\alpha_{3} \beta_{4}}^{\beta_{3} \beta_{4}} R_{\alpha_{5} \alpha_{6}}^{\beta_{5} \beta_{6}}, \\
B_{2}^{\mu \nu} & =-12 \sqrt{g} \varepsilon^{\mu \alpha_{2} \nu \alpha_{4} \alpha_{5} \alpha_{6}} \varepsilon_{\beta_{1} \ldots \beta_{6}} \delta \Gamma_{\gamma \alpha_{2}}^{\beta_{1}} \cdot g^{\gamma \beta_{2}} \delta \Gamma_{\rho \alpha_{4}}^{\beta_{3}} g^{\rho \beta_{4}} R_{\alpha_{5} \alpha_{6}}^{\beta_{5} \beta_{6}}, \\
B_{3}^{\mu \nu \rho} & =8 \sqrt{g} \varepsilon^{\mu \alpha_{2} \nu \alpha_{4} \rho \alpha_{6}} \varepsilon_{\beta_{1} \ldots \beta_{6}} \delta \Gamma_{\gamma \alpha_{2}}^{\beta_{1}} \cdot g^{\gamma \beta_{2}} \delta \Gamma_{\mu \alpha_{4}}^{\beta_{3}} g^{\mu \beta_{4}} \delta \Gamma_{\nu \alpha_{6}}^{\beta^{5}} g^{\nu \beta_{6}},
\end{aligned}
$$

где $B_{1}^{\mu}, B_{2}^{\mu \nu}$ и $B_{3}^{\mu \nu \rho}-$ соответственно 1-, 2- и 3-коциклы. 
Полезно привести выражение для $E_{6}$ в терминах тензоров Римана и Вейля:

$$
\begin{aligned}
E_{6} & =8 \sqrt{g}\left(2 I_{1}-8 I_{2}-24 I_{3}+3 I_{4}+24 I_{5}+16 I_{6}-12 I_{7}+I_{8}\right)= \\
& =8 \sqrt{g}\left(2 J_{1}-8 J_{2}-6 J_{3}+\frac{6}{5} J_{4}+3 J_{5}+\frac{3}{2} I_{6}-\frac{27}{20} I_{7}+\frac{21}{100} I_{8}\right),
\end{aligned}
$$

где использован следующий базис скаляров, построенных из тензоров Римана и Вейля:

$$
\begin{array}{lll}
I_{1}=R_{\mu \nu}^{\alpha \beta} R_{\lambda \sigma}^{\mu \nu} R_{\alpha \beta}^{\lambda \sigma}, & J_{1}=C_{\mu \nu}^{\alpha \beta} C_{\lambda \sigma}^{\mu \nu} C_{\alpha \beta}^{\lambda \sigma}, \\
I_{2}=R_{\mu \nu}^{\lambda \alpha} R_{\lambda \rho}^{\mu \sigma} R_{\alpha \sigma}^{\nu \rho}, & J_{2}=C_{\mu \nu}^{\lambda \alpha} C_{\lambda \rho}^{\mu \sigma} C_{\alpha \sigma}^{\nu \rho}, \\
I_{3}=R_{\mu}^{\nu} R_{\rho \sigma}^{\mu \lambda} R_{\nu \lambda}^{\rho \sigma}, & J_{3}=R_{\mu}^{\nu} C_{\rho \sigma}^{\mu \lambda} C_{\nu \lambda,}^{\rho \sigma}, \\
I_{4}=R R_{\rho \sigma}^{\mu \nu} R_{\mu \nu}^{\rho \sigma}, & J_{4}=R C_{\rho \sigma}^{\mu \nu} C_{\mu \nu}^{\rho \sigma}, \\
I_{5}=R_{\nu}^{\mu} R_{\rho}^{\sigma \sigma} R_{\mu \sigma}^{\nu \rho}, & J_{5}=R_{\nu}^{\mu} R_{\rho}^{\sigma} C_{\mu \sigma}^{\nu \rho}, \\
I_{6}=R^{\mu \nu} R_{\mu \sigma} R_{\nu}^{\sigma}, & I_{8}=R^{3}, \\
I_{7}=R R^{\mu \nu} R_{\mu \nu}, & K=C_{\mu \nu}^{\alpha \beta} \Delta C_{\alpha \beta}^{\mu \nu} .
\end{array}
$$

Используя определение тензора Вейля, мы легко получим формулы перехода от одного базиса полиномов от тензоров Римана к другому, в котором все тензоры Римана заменены тензорами Вейля:

$$
\begin{gathered}
I_{1}=J_{1}+3 J_{3}-\frac{3}{10} J_{4}+\frac{3}{2} J_{5}+\frac{1}{4} I_{6}+\frac{3}{40} I_{7}-\frac{3}{200} I_{8}, \\
I_{2}=J_{2}-\frac{3}{2} J_{3}+\frac{3}{20} J_{4}-\frac{3}{8} I_{6}+\frac{3}{16} I_{7}-\frac{7}{400} I_{8}, \\
I_{3}=J_{3}+J_{5}+\frac{1}{4} I_{6}+\frac{7}{40} I_{7}-\frac{1}{40} I_{8}, \\
I_{4}=J_{4}+I_{7}-\frac{1}{10} I_{8}, \\
I_{5}=J_{5}+\frac{11}{20} I_{7}-\frac{1}{2} I_{6}-\frac{1}{20} I_{8}, \\
C^{\nu \alpha \beta \gamma} C_{\alpha \beta \gamma}^{\rho}=R^{\nu \alpha \beta \gamma} R_{\alpha \beta \gamma}^{\rho}-R^{\nu \alpha \rho \beta} R_{\alpha \beta}-\frac{3}{4} R^{\nu \alpha} R_{\alpha}^{\rho}+ \\
\quad+\frac{1}{8} R^{\alpha \beta} R_{\alpha \beta} g^{\nu \rho}+\frac{1}{4} R^{\nu \rho} R-\frac{1}{40} g^{\nu \rho} R^{2}, \\
C^{\nu \alpha \beta \gamma} C_{\nu \alpha \beta \gamma}=R^{\nu \alpha \beta \gamma} R_{\nu \alpha \beta \gamma}-R^{\alpha \beta} R_{\alpha \beta}+\frac{1}{10} R^{2} .
\end{gathered}
$$

Следующий шаг состоит в выполнении аналогичных преобразований для остальных ко- 
циклов:

$$
\begin{aligned}
B_{1}^{\mu}= & 24 \sqrt{g}\left[\nabla^{\mu} \delta \Omega \cdot\left(R_{\nu \rho}^{\alpha \beta} R_{\alpha \beta}^{\nu \rho}-4 R^{\alpha \beta} R_{\alpha \beta}+R^{2}\right)-\right. \\
& \left.-4 \nabla_{\rho} \delta \Omega \cdot\left(R^{\mu \alpha \beta} R_{\alpha \beta \gamma}^{\rho}-2 R^{\mu \alpha \rho \beta} R_{\alpha \beta}-2 R^{\mu \alpha} R_{\alpha}^{\rho}+R^{\mu \rho} R\right)\right], \\
B_{1}^{\mu}= & 4 \sqrt{g}\left[\nabla^{\mu} \delta \Omega\left(C^{\alpha \beta \nu \rho} C_{\alpha \beta \nu \rho}-\frac{3}{2} R^{\alpha \beta} R_{\alpha \beta}+\frac{3}{5} R^{2}\right)+\right. \\
& \left.+\nabla_{\rho} \delta \Omega \cdot\left(-4 C^{\mu \alpha \beta \gamma} C_{\alpha \beta \gamma}^{\rho}+4 C^{\mu \alpha \rho \beta} R_{\alpha \beta}+3 R^{\mu \alpha} R_{\alpha}^{\rho}-\frac{9}{5} R^{\mu \rho} R\right)\right], \\
B_{2}^{\mu \nu}= & -96 \sqrt{g}\left(R_{\alpha \beta}^{\mu \nu} \nabla^{\alpha} \delta \Omega \cdot \nabla^{\beta} \delta \Omega+2 R_{\alpha}^{\mu} \nabla^{\nu} \delta \Omega \cdot \nabla^{\alpha} \delta \Omega-\right. \\
& \left.-2 R_{\alpha}^{\nu} \nabla^{\mu} \delta \Omega \cdot \nabla^{\alpha} \delta \Omega+R \nabla^{\mu} \delta \Omega \cdot \nabla^{\nu} \delta \Omega\right), \\
B_{2}^{\mu \nu}= & -48 \sqrt{g}\left(2 C^{\mu \nu \rho \pi} \nabla_{\rho} \delta \Omega \cdot \nabla_{\pi} \delta \Omega+3 R_{\alpha}^{\mu} \nabla^{\nu} \delta \Omega \cdot \nabla^{\alpha} \delta \Omega-\right. \\
& \left.-3 R_{\alpha}^{\nu} \nabla^{\mu} \delta \Omega \cdot \nabla^{\alpha} \delta \Omega+\frac{9}{5} R \nabla^{\mu} \delta \Omega \cdot \nabla^{\nu} \delta \Omega\right), \\
B_{3}^{\mu \nu \rho}= & -288 \sqrt{g} \nabla^{\mu} \delta \Omega \cdot \nabla^{\nu} \delta \Omega \cdot \nabla^{\rho} \delta \Omega .
\end{aligned}
$$

Рассмотрим теперь аналогичную процедуру для конформной аномалии второго типа (В) из геометрической классификации [2], а затем сравним соответствующие коциклы. Аномалия последнего типа состоит из двух тензоров Вейля и одного лапласиана и имеет вид

$$
\begin{aligned}
A_{3}= & \sqrt{g}\left(C_{\rho \sigma}^{\mu \nu} \Delta C_{\mu \nu}^{\rho \sigma}+2 C^{\mu \nu \rho \alpha} C_{\mu \nu \rho \alpha} R_{\alpha}^{\beta}-3 C_{\mu \nu \rho \sigma} R^{\mu \rho} R^{\nu \sigma}-\right. \\
& \left.-\frac{3}{2} R^{\nu \sigma} R_{\sigma \alpha} R_{\nu}^{\alpha}+\frac{27}{20} R^{\mu \nu} R_{\mu \nu} R-\frac{21}{100} R^{3}\right)
\end{aligned}
$$

Оставляя громоздкие вычисления, получаем следующее уравнение спуска:

$$
\begin{aligned}
\delta A_{3} & =\widetilde{\nabla}_{\mu} A_{1}^{\mu}, \\
\delta A_{1}^{\mu} & =\widetilde{\nabla}_{\nu} A_{2}^{\mu \nu}, \\
\delta A_{2}^{\mu \nu} & =\widetilde{\nabla}_{\pi} A_{3}^{\mu \nu \pi}, \\
\delta A_{3}^{\mu \nu \pi} & =0
\end{aligned}
$$

где

$$
\begin{aligned}
A_{1}^{\mu}= & \sqrt{g}\left(4 R^{\rho \sigma \nu \mu} R_{\rho \sigma \nu \pi} \nabla^{\pi} \delta \Omega-16 R^{\rho \mu \nu \pi} R_{\rho \nu} \nabla_{\pi} \delta \Omega+\right. \\
& +8 R^{\nu \rho} R_{\nu \rho} \nabla^{\mu} \delta \Omega-18 R^{\sigma \mu} R_{\sigma \pi} \nabla^{\pi} \delta \Omega+ \\
& \left.+10 R^{\mu \pi} R \cdot \nabla_{\pi} \delta \Omega-\frac{5}{2} R^{2} \nabla^{\mu} \delta \Omega\right), \\
A_{2}^{\mu \rho}= & 12 \sqrt{g}\left(R^{\mu \rho \nu \pi} \nabla_{\nu} \delta \Omega \cdot \nabla_{\pi} \delta \Omega+2 R^{\mu \nu} \nabla^{\rho} \delta \Omega \cdot \nabla_{\nu} \delta \Omega-\right. \\
& \left.-2 R^{\rho \nu} \nabla^{\mu} \delta \Omega \cdot \nabla_{\nu} \delta \Omega+R \nabla^{\mu} \delta \Omega \cdot \nabla^{\rho} \delta \Omega\right), \\
A_{3}^{\mu \nu \pi}= & 36 \sqrt{g} \nabla^{\mu} \delta \Omega \cdot \nabla^{\nu} \delta \Omega \cdot \nabla^{\pi} \delta \Omega .
\end{aligned}
$$


Величины $A_{1}^{\mu}, A_{2}^{\mu \nu}, A_{3}^{\mu \nu \pi}$ являются 1-, 2-, 3-коциклами группы Вейля, соответственно. Как и в предыдушем случае, $A_{1}^{\mu}$ и $A_{2}^{\mu \rho}$ представляются в виде

$$
\begin{aligned}
A_{1}^{\mu}= & \sqrt{g}\left[\nabla^{\mu} \delta \Omega \cdot\left(\frac{9}{2} R^{\nu \rho} R_{\nu \rho}-\frac{9}{5} R^{2}\right)+\nabla_{\rho} \delta \Omega \cdot\left(4 C^{\mu \pi \nu \sigma} C_{\pi \nu \sigma}^{\rho}-\right.\right. \\
& \left.\left.-12 C^{\mu \pi \rho \sigma} R_{\pi \sigma}-9 R^{\mu \sigma} R_{\sigma}^{\rho}+\frac{27}{5} R^{\mu \rho} R\right)\right], \\
A_{2}^{\mu \rho}= & 6 \sqrt{g}\left[2 C^{\mu \rho \nu \pi} \nabla_{\nu} \delta \Omega \cdot \nabla_{\pi} \delta \Omega-3 R^{\rho \nu} \nabla^{\mu} \delta \Omega \cdot \nabla_{\nu} \delta \Omega+\right. \\
& \left.+3 R^{\mu \nu} \nabla^{\rho} \delta \Omega \cdot \nabla_{\nu} \delta \Omega+\frac{9}{5} R \nabla^{\mu} \delta \Omega \cdot \nabla^{\rho} \delta \Omega\right] .
\end{aligned}
$$

Из сравнения (29) и (32) непосредственно следует, что

$$
\frac{1}{8} B_{1}^{\mu}+A_{1}^{\mu}=\sqrt{g}\left(3 \nabla^{\mu} \delta \Omega \cdot C^{\alpha \beta \nu \rho} C_{\alpha \beta \nu \rho}-8 \nabla_{\rho} \delta \Omega \cdot C^{\mu \alpha \beta \gamma} C_{\alpha \beta \gamma}^{\rho}\right) .
$$

Последнее выражение легко интегрируется:

$$
\frac{1}{8} B_{1}^{\mu}+A_{1}^{\mu}=\delta\left(-3 \sqrt{g} C_{\gamma \delta}^{\alpha \beta} \nabla^{\mu} C_{\alpha \beta}^{\gamma \delta}-\frac{16}{3} \sqrt{g} C_{\alpha \beta}^{\mu \nu} \nabla^{\rho} C_{\rho \nu}^{\alpha \beta}\right) .
$$

Тогда из первых уравнений спуска для аномалий типов (А) и (В) имеем

$$
\delta\left(\frac{1}{8} E_{6}+A_{3}\right)=\sqrt{g} \nabla_{\mu} \frac{1}{\sqrt{g}}\left(\frac{1}{8} B_{1}^{\mu}+A_{1}^{\mu}\right)
$$

или, что то же самое,

$$
\delta\left(\frac{1}{8} E_{6}+A_{3}+\sqrt{g} \nabla_{\mu}\left(3 C^{\alpha \beta \gamma \delta} \nabla^{\mu} C_{\alpha \beta \gamma \delta}+\frac{16}{3} C^{\mu \nu \alpha \beta} \nabla_{\rho} C^{\rho}{ }_{\nu \alpha \beta}\right)\right)=0 .
$$

С помощью представления (26) прямым вычислением можно убедиться в инвариантности выражения (36) относительно вейлевских преобразований. Действительно, посколькy

$$
\frac{1}{8} E_{6}+A_{3}=\sqrt{g}\left(2 J_{1}-8 J_{2}-4 J_{3}+\frac{6}{5} J_{4}+K\right)
$$

$$
\begin{aligned}
\delta \sqrt{g} J_{3}= & 2 \sqrt{g} C_{\rho \sigma}^{\mu \lambda} C_{\nu \lambda}^{\rho \sigma} \nabla^{\nu} \nabla_{\mu} \delta \Omega+\frac{1}{2} \sqrt{g} C_{\rho \sigma}^{\mu \lambda} C_{\mu \lambda}^{\rho \sigma} \nabla_{\alpha} \nabla^{\alpha} \delta \Omega, \\
\delta \sqrt{g} J_{4}= & 5 \sqrt{g} C_{\rho \sigma}^{\mu \nu} C_{\mu \nu}^{\rho \sigma} \nabla_{\alpha} \nabla^{\alpha} \delta \Omega, \\
\delta \sqrt{g} K= & -4 \sqrt{g} C^{\mu \nu \rho \sigma} \nabla_{\mu} \delta \Omega \cdot \nabla^{\pi} C_{\pi \nu \rho \sigma}+4 \sqrt{g} C^{\mu \nu \rho \sigma} \nabla_{\mu} C_{\pi \nu \rho \sigma} \cdot \nabla^{\pi} \delta \Omega- \\
& -\sqrt{g} C^{\mu \nu \rho \sigma} C_{\mu \nu \rho \sigma} \nabla^{\alpha} \nabla_{\alpha} \delta \Omega,
\end{aligned}
$$

можно немедленно получить следуюшие соотношения:

$$
\begin{aligned}
& \delta\left(\frac{1}{8} E_{6}+A_{3}\right)=3 \sqrt{g} C_{\rho \sigma}^{\mu \nu} C_{\mu \nu}^{\rho \sigma} \nabla_{\alpha} \nabla^{\alpha} \delta \Omega-8 \sqrt{g} C_{\rho \sigma}^{\mu \lambda} C_{\nu \lambda}^{\rho \sigma} \nabla^{\nu} \nabla_{\mu} \delta \Omega- \\
& \quad-4 \sqrt{g} C^{\mu \nu \rho \sigma} \nabla^{\pi} C_{\pi \nu \rho \sigma} \nabla_{\mu} \delta \Omega+4 \sqrt{g} C^{\mu \nu \rho \sigma} \nabla_{\mu} C_{\pi \nu \rho \sigma} \nabla^{\pi} \delta \Omega \\
& \sqrt{g} \nabla_{\mu} \frac{1}{\sqrt{g}}\left(\frac{1}{8} B_{1}^{\mu}+A_{1}^{\mu}\right)=3 \sqrt{g} C_{\nu \rho}^{\alpha \beta} C_{\alpha \beta}^{\nu \rho} \nabla^{\mu} \nabla_{\mu} \delta \Omega- \\
& \quad-8 \sqrt{g} C_{\beta \gamma}^{\mu \alpha} C_{\rho \alpha}^{\beta \gamma} \nabla_{\mu} \nabla^{\rho} \delta \Omega+6 \sqrt{g} C_{\nu \rho}^{\alpha \beta} \nabla_{\mu} C_{\alpha \beta}^{\nu \rho} \cdot \nabla^{\mu} \delta \Omega- \\
& -8 \sqrt{g} \nabla_{\mu} C^{\mu \alpha \beta \gamma} \cdot C_{\alpha \beta \gamma}^{\rho} \nabla_{\rho} \delta \Omega-8 \sqrt{g} C^{\mu \alpha \beta \gamma} \nabla_{\mu} C_{\alpha \beta \gamma}^{\rho} \nabla_{\rho} \delta \Omega .
\end{aligned}
$$


На первый взгляд разность двух соотношений (39) не равна нулю, а дает

$$
\begin{aligned}
\delta\left(\frac{1}{8} E_{6}+A_{3}\right)-\sqrt{g} \nabla_{\mu} \frac{1}{\sqrt{g}}\left(\frac{1}{8} B_{1}^{\mu}+A_{1}^{\mu}\right)= \\
=-6 \sqrt{g} \nabla^{\mu} \delta \Omega \cdot C_{\nu \rho}^{\alpha \beta} \nabla_{\mu} C_{\alpha \beta}^{\nu \rho}+4 \sqrt{g} \nabla_{\rho} \delta \Omega \cdot \nabla_{\mu} C_{\alpha \beta \gamma}^{\mu} \cdot C^{\rho \alpha \beta \gamma}+ \\
\quad+12 \sqrt{g} \nabla_{\rho} \delta \Omega \cdot C^{\mu \alpha \beta \gamma} \nabla_{\mu} C^{\rho}{ }_{\alpha \beta \gamma} \equiv-6 G_{1}+4 G_{2}+12 G_{3} .
\end{aligned}
$$

Однако благодаря равенствам

$$
\begin{aligned}
G_{1}= & \sqrt{g} \nabla^{\mu} \delta \Omega \cdot C_{\nu \rho}^{\alpha \beta} \cdot \nabla_{\mu} C_{\alpha \beta}^{\nu \rho}=2 \sqrt{g} \nabla^{\mu} \delta \Omega \cdot C^{\alpha \beta \nu \rho} \nabla_{\alpha} R_{\mu \beta \nu \rho}, \\
G_{2}= & \sqrt{g} \nabla_{\rho} \delta \Omega \cdot C^{\rho}{ }_{\alpha \beta \gamma} \nabla_{\mu} C^{\mu \alpha \beta \gamma}=-\frac{3}{2} \sqrt{g} \nabla_{\rho} \delta \Omega \cdot C_{\beta \gamma \alpha}{ }^{\rho} \nabla^{\beta} R^{\gamma \alpha}, \\
G_{3}= & \sqrt{g} \nabla_{\rho} \delta \Omega \cdot C^{\mu \alpha \beta \gamma} \nabla_{\mu} C^{\rho}{ }_{\alpha \beta \gamma}=\sqrt{g} \nabla_{\rho} \delta \Omega \cdot C^{\mu \alpha \beta \gamma} \nabla_{\mu} R_{\alpha \beta \gamma}^{\rho}+ \\
& +\frac{1}{2} \sqrt{g} \nabla_{\beta} \delta \Omega \cdot C^{\mu \alpha \gamma \beta} \cdot \nabla_{\mu} R_{\alpha \gamma}
\end{aligned}
$$

получаем нетривиальное тождество

$$
-6 G_{1}+4 G_{2}+12 G_{3}=0
$$

Таким образом, мы проверили инвариантность соотношения (37) двумя различными способами: во-первых, используя уравнение спуска, во-вторых, непосредственными вычислениями, используя нетривиальное тождество (42). Сравнивая выражения (40) и (42), немедленно получаем

$$
\begin{aligned}
& \delta\left(\sqrt { g } \left(-4 J_{3}+\frac{6}{5} J_{4}+4 K+3 \nabla_{\mu} C_{\gamma \delta}^{\alpha \beta} \cdot \nabla^{\mu} C_{\alpha \beta}^{\gamma \delta}+\right.\right. \\
& \left.\left.\quad+\frac{16}{3} \nabla_{\mu} C^{\mu \nu \alpha \beta} \cdot \nabla_{\rho} C_{\nu \alpha \beta}^{\rho}+\frac{16}{3} C^{\mu \nu \alpha \beta} \nabla_{\mu} \nabla_{\rho} C^{\rho}{ }_{\nu \alpha \beta}\right)\right)
\end{aligned}
$$

Соотношение (37) - пример нетривиального конформного (вейлевского) инварианта, который состоит из двух тензоров Вейля и двух ковариантных производных.

Теперь рассмотрим следствия этих вычислений и опишем возможные обобшения последних. Первое заключение, следуюшее из соотношения (40), - эквивалентность аномалий типов $(\mathrm{A})$ и $(\mathrm{B})$ в слабом смысле. Второе заключение состоит в том, что подход, основанный на уравнении спуска, дает систематический способ обобщения этих результатов на высшие размерности $D=8,10, \ldots$ и т.д. Используя полученные результаты, приведем другой тип конформной аномалии, который эквивалентен $E_{6}$ в когомологическом смысле, но отличается с геометрической точки зрения. Для этого мы рассмотрим выражение для $B_{3}^{\mu \nu \rho}$ и попытаемся интегрировать уравнения спуска снизу вверх:

$$
\widetilde{\nabla}_{\rho} \widetilde{B}_{3}^{\mu \nu \rho}=\delta \bar{B}_{2}^{\mu \nu}
$$

где

$$
\bar{B}_{2}^{\mu \nu}=-144 \sqrt{g}\left\{R_{\rho}^{\mu} \nabla^{\nu} \delta \Omega \cdot \nabla^{\rho} \delta \Omega-R_{\rho}^{\nu} \nabla^{\mu} \delta \Omega \cdot \nabla^{\rho} \delta \Omega+\frac{3}{5} R \nabla^{\mu} \delta \Omega \cdot \nabla^{\nu} \delta \Omega\right\} .
$$


Заметим, что 2-коцикл $\bar{B}_{2}^{\mu \nu}$ не совпадает с ранее полученными выражениями для $B_{2}^{\mu \nu}$ и $A_{2}^{\mu \nu}$. Разность последних, как нетрудно проверить, следующая:

$$
B_{2}^{\mu \nu}-\bar{B}_{2}^{\mu \nu}=-96 \sqrt{g} C_{\alpha \beta}^{\mu \nu} \nabla^{\alpha} \delta \Omega \cdot \nabla^{\beta} \delta \Omega,
$$

и является вейлевским инвариантом, как это и следует из уравнения спуска. Оставляя подробности сложных вычислений, мы восстанавливаем обшую форму аномалии, соответствуюшей 2-коциклу $\bar{B}_{2}^{\mu \nu}$, с точностью до числовых констант:

$$
\begin{aligned}
D= & \sqrt{g}\left(\alpha_{1} \nabla^{\mu} R \cdot \nabla_{\mu} R+\alpha_{2} R \Delta R+\alpha_{3} R^{\mu \nu} \nabla_{\mu} \nabla_{\nu} R+\alpha_{4} R R^{\mu \nu} R_{\mu \nu}+\right. \\
& +\alpha_{5} \nabla^{\nu} R_{\rho}^{\mu} \cdot \nabla_{\mu} R_{\nu}^{\rho}+\alpha_{6} R_{\rho}^{\mu} R_{\nu}^{\rho} R_{\mu}^{\nu}+\alpha_{7} R^{\mu \nu} \Delta R_{\mu \nu}+ \\
& \left.+\alpha_{8} \nabla_{\lambda} R^{\mu \nu} \nabla^{\lambda} R_{\mu \nu}+\alpha_{9} R_{\rho}^{\nu} R_{\nu}^{\rho} R+\alpha_{10} R^{3}+\alpha_{11} \Delta \Delta R\right)+(\ldots),
\end{aligned}
$$

где точки обозначают слагаемые, включающие тензоры Римана. И снова этот тип аномалий по геометрической классификации отличается от ранее найденных выражений $(25)$ и (30), но эквивалентен аномалии $E_{6}$ в когомологическом смысле.

\section{4. КОНФОРМНО-ИНВАРИАНТНЫЕ ОПЕРАТОРЫ В РАЗМЕРНОСТИ $D=6$}

Обсудим проблему построения эффективного гравитационного действия в размерности $D=6$ при помоши уже известных выражений для конформной аномалии. Можно попытаться непосредственно обобщить результаты работы [8], где построено нелокальное гравитационное действие в размерности $D=4$, хотя на этом пути имеются определенные трудности, которые показывают сушественное различие между случаями $D=4$ и $D=6$. Процедура построения действия, предложенная в работе [8], основана на существовании конформно-инвариантного оператора 4-го порядка (включающего оператор $\Delta^{2}$ как слагаемое) и особой структуры конформной аномалии в размерности $D=4$. Покажем, что в размерности $D=6$ конформно-инвариантный оператор 6-го порядка (включающий $\Delta^{3}$ как слагаемое), построенный из тензоров Римана, Риччи, скалярной кривизны и ковариантных производных, не существует. Доказательство этого утверждения основано на уравнении спуска и получается следующим образом. Предположим противное и допустим, что такой оператор построен. Тогда имеем

$$
P=\sqrt{g} \Delta^{3}+D
$$

и

где введен оператор

$$
\delta \sqrt{g} \Delta^{3}=\sqrt{g} \nabla_{\nu} \frac{1}{\sqrt{g}} \delta\left(\sqrt{g} \nabla^{\nu} \Delta \Delta\right)
$$

$$
D=\sqrt{g} \nabla_{\nu} D^{\nu}=\widetilde{\nabla}_{\nu} \sqrt{g} D^{\nu} .
$$

Учитывая инвариантность $P$, получим

$$
\delta P=\widetilde{\nabla}_{\nu} \delta\left(\sqrt{g} \nabla^{\nu} \Delta \Delta\right)+\widetilde{\nabla}_{\nu} \delta \sqrt{g} D^{\nu}=0,
$$

так что имеем

$$
\delta\left(\sqrt{g} \nabla^{\nu} \Delta \Delta\right)+\delta\left(\sqrt{g} D^{\nu}\right)=\widetilde{\nabla}_{\mu} F_{1}^{\nu \mu},
$$


где $F_{1}^{\mu \nu} \equiv-F_{1}^{\nu \mu}$. Остальная часть доказательства заключается в анализе уравнения (52). Во-первых, выражение для $F_{1}^{\mu \nu}$ не может быть произвольным, а должно удовлетворять следуюшим соотношениям уравнения спуска, т.е.

$$
\begin{aligned}
\delta F_{1}^{\nu \mu} & =\widetilde{\nabla}_{\rho} F_{2}^{\nu \mu \rho}, \\
\delta F_{2}^{\nu \mu \rho} & =0 .
\end{aligned}
$$

Непосредственное вычисление показывает, что

$$
\begin{aligned}
\delta\left(\sqrt{g} \nabla^{\nu} \Delta \Delta\right)=\sqrt{g} \nabla_{\sigma} F_{1}^{\nu \sigma}+\delta\left(2 \sqrt{g} R^{\nu \mu} \nabla_{\mu} \Delta+2 \sqrt{g} R^{\mu \sigma} \nabla^{\nu} \nabla_{\mu} \nabla_{\sigma}-\sqrt{g} R \nabla^{\nu} \Delta\right)+ \\
+\left(-\sqrt{g} \nabla^{\nu} \Delta \delta \Omega \cdot \Delta+2 \sqrt{g} \nabla^{\sigma} \Delta \delta \Omega \cdot \nabla^{\nu} \nabla_{\sigma}+4 \sqrt{g} \nabla^{\nu} \nabla^{\lambda} \nabla^{\sigma} \delta \Omega \cdot \nabla_{\lambda} \nabla_{\sigma}-\right. \\
\quad-10 \sqrt{g} R^{\nu \mu} \nabla^{\rho} \delta \Omega \cdot \nabla_{\mu} \nabla_{\rho}+6 \sqrt{g} R^{\mu \sigma} \nabla^{\nu} \delta \Omega \cdot \nabla_{\mu} \nabla_{\sigma}+ \\
+4 \sqrt{g} R^{\nu \mu} \nabla_{\mu} \delta \Omega \cdot \Delta-2 \sqrt{g} R^{\sigma \rho} \nabla_{\rho} \delta \Omega \cdot \nabla_{\sigma} \nabla^{\nu}+ \\
\left.+\frac{12}{5} \sqrt{g} R \nabla^{\lambda} \delta \Omega \cdot \nabla_{\lambda} \nabla^{\nu}-\frac{12}{5} \sqrt{g} R \nabla^{\nu} \delta \Omega \cdot \Delta\right)+(\ldots)
\end{aligned}
$$

где $F_{1}^{\mu \nu}$ имеет вид

$$
\begin{aligned}
F_{1}^{\mu \nu}= & 2 \sqrt{g}\left(\nabla^{\mu} \delta \Omega \cdot \nabla^{\nu} \Delta-\nabla^{\nu} \delta \Omega \cdot \nabla^{\mu} \Delta\right)+ \\
& +4 \sqrt{g}\left(R^{\nu \rho} \nabla^{\mu} \delta \Omega \cdot \nabla_{\rho}-R^{\mu \rho} \nabla^{\nu} \delta \Omega \cdot \nabla_{\rho}\right)+ \\
& +2 \sqrt{g}\left(R^{\mu \rho} \nabla_{\rho} \delta \Omega \cdot \nabla^{\nu}-R^{\nu \rho} \nabla_{\rho} \delta \Omega \cdot \nabla^{\mu}\right)+ \\
& +\frac{7}{5} \sqrt{g}\left(R \nabla^{\nu} \delta \Omega \cdot \nabla^{\mu}-R \cdot \nabla^{\mu} \delta \Omega \cdot \nabla^{\nu}\right),
\end{aligned}
$$

а точки обозначают операторы первого порядка. Дальнейшие вычисления показьвают, что $F_{1}^{\mu \nu}$ действительно удовлетворяет уравнению спуска (53), а $F_{2}^{\mu \nu \rho}$ имеет форму

$$
F_{2}^{\mu \nu \rho}=4 \sqrt{g}\left(\nabla^{\mu} \delta \Omega \cdot \nabla^{\nu} \delta \Omega \cdot \nabla^{\rho}+\nabla^{\nu} \delta \Omega \cdot \nabla^{\rho} \delta \Omega \cdot \nabla^{\mu}+\nabla^{\rho} \delta \Omega \cdot \nabla^{\mu} \delta \Omega \cdot \nabla^{\nu}\right) .
$$

Следуюший шаг заключается в построении таких операторов 2-го порядка, состояших из тензоров Римана (Риччи, скалярной кривизны) и ковариантных производных, вариации которых сокрашают операторную часть 2-го порядка в выражении (63). Однако имеется только ограниченное число операторов 2-го порядка, а именно:

$$
\sqrt{g} \nabla^{\nu} R \Delta ; \quad \sqrt{g} \nabla^{\mu} R \nabla^{\nu} \nabla_{\mu} ; \quad \sqrt{g} \nabla^{\nu} R^{\mu \rho} \cdot \nabla_{\mu} \nabla_{\rho} ; \quad \sqrt{g} \nabla^{\mu} R^{\nu \rho} \nabla_{\mu} \nabla_{\rho}
$$

(все остальные операторы, которые могут быть построены из тензоров Римана благодаря тождеству Бианки и свойствам симметрии, приводятся к отмеченным выше). Выполняя вычисления, находим

$$
\begin{aligned}
\delta\left(A \sqrt{g} \nabla^{\nu} R \cdot \Delta+B \sqrt{g} \nabla^{\mu} R \cdot \nabla^{\nu} \nabla_{\mu}+C \sqrt{g} \nabla^{\nu} R^{\mu \rho} \nabla_{\mu} \nabla_{\rho}+F \sqrt{g} \nabla^{\mu} R^{\nu \rho} \nabla_{\mu} \nabla_{\rho}\right)= \\
=\sqrt{g}\left(\left(5 A+\frac{C}{2}\right) \nabla^{\nu} \Delta \delta \Omega \cdot \Delta+\left(5 B+\frac{F}{2}\right) \nabla^{\mu} \Delta \delta \Omega \cdot \nabla^{\nu} \nabla_{\mu}+\right. \\
\quad+(2 C+2 F) \nabla^{\mu} \nabla^{\nu} \nabla^{\rho} \delta \Omega \cdot \nabla_{\mu} \nabla_{\rho}+\left(C+\frac{F}{2}\right) R^{\sigma \rho} \nabla_{\sigma} \delta \Omega \cdot \nabla^{\nu} \nabla_{\rho}- \\
\quad-(C+F) R^{\nu \rho} \nabla^{\mu} \delta \Omega \cdot \nabla_{\mu} \nabla_{\rho}-\left(C+\frac{F}{2}\right) R^{\mu \rho} \nabla^{\nu} \delta \Omega \cdot \nabla_{\mu} \nabla_{\rho}- \\
\left.-B \cdot R \nabla^{\mu} \delta \Omega \cdot \nabla_{\mu} \nabla^{\nu}-A R \nabla^{\nu} \delta \Omega \cdot \Delta+\frac{F}{2} R^{\nu \mu} \nabla_{\mu} \delta \Omega \cdot \Delta\right)+(\ldots)
\end{aligned}
$$


где точки обозначают операторы 1-го порядка, а константы $A, B$ и $C$ определяются из сравнения (54) и (58). Тогда для четырех констант мы получаем 9 уравнений (количество операторов 2-го порядка в (54)). Эта система уравнений переопределена и не имеет решения. Последнее наблюдение завершает доказательство утверждения об отсутствии конформно-инвариантного оператора 6-го порядка в размерности $D=6$. Таким образом, структура конформной аномалии в размерности $D=4$ существенным образом отличается от той, которая имеется в размерности $D=6$, и эффективное гравитационное действие в этом случае не может быть построено в полной аналогии с тем подходом, который развивается в работе [8].

\section{5. ЗАКЛЮЧЕНИЕ}

Суммируем основные результаты, полученные в этой работе. Во-первых, предлагается новый подход к проблеме классификаций конформных аномалий в высших размерностях. Продемонстрировано преимущество когомологической классификации, основанной на технике уравнений спуска, по сравнению с геометрической. Найден новый конформный инвариант, который используется при доказательстве эквивалентности аномалий типов $(\mathrm{A})$ и (В). Для построения эффективного действия исследованы конформно-инвариантные операторы в размерности $D=6$. В этом случае мы имеем отрицательный ответ в отношении сушествования оператора подобного типа, который означает, что структуры конформных аномалий сушественным образом различаются в случае размерностей $D=4$ и $D=6$. Возможные пути решения этих проблем при $D=6$ исследуются.

\section{Список литературы}

[1] S. Deser, M. J. Duff, C. J. Isham. Nucl. Phys. B. 1976. V. 111. P. 173; M. J. Duff. Nucl. Phys. B. 1977. V. 125. P. 334; D. M. Capper, M. J. Duff. Nuovo Cimento. 1974. V. 23. P. 173.

[2] S. Deser, A. Schwimer. Phys. Lett. B. 1993. V. 309. P. 279.

[3] L. Bonora, P. Pasti, M. Tonin. Phys. Lett. B. 1984. V. 149. P. 346; L. Bonora, P. Cotta-Ramusino, C. Reina. Phys. Lett. B. 1983. V. 126. P. 305.

[4] M. Bregola, P. Pasti, L. Bonora. Class. Quantum. Gravit. 1986. V. 3. P. 635.

[5] L. D. Faddeev. Phys. Lett. B. 1984. V. 145. Р. 81; Л. Д. Фаддеев, С. Л. Шаташвили. ТМФ. 1984. T. 60. № 2. C. 206.

[6] H. Cartan, S. Eilenberg. Homological Algebra. Princeton: Princ. University Press, 1956; Д. Б. Фукс. Когомологии бесконечномерных алгебр Ли. М.: Наука, 1984.

[7] B. Zumino. Nucl. Phys. B. 1985. V. 253. P. 177; W. Bardin, B. Zumino. Nucl. Phys. B. 1984. V. 244. P. 421; J. Wess, B. Zumino. Phys. Lett. B. 1971. V. 37. P. 95.

[8] R. J. Rigert. Phys. Lett. B. 1984. V. 134. P. 56. 